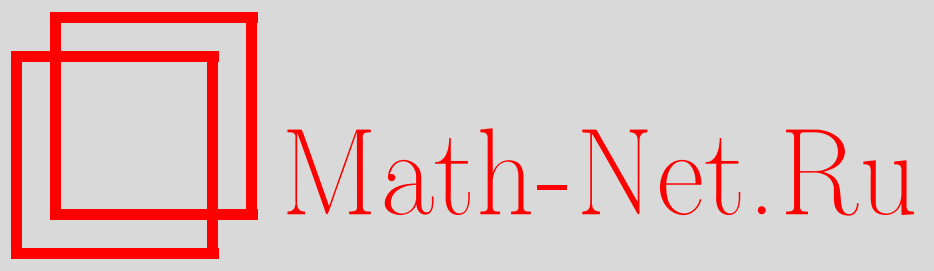

Ф. М. Мухамедов, Ч. Пах, Х. Джамиль, Основные состояния и фазовые переходы $\lambda$-модели на дереве Кэли, TMФ, 2018, том 194, номер 2, 304-319

DOI: https://doi.org/10.4213/tmf9309

Использование Общероссийского математического портала Math-Net.Ru подразумевает, что вы прочитали и согласны с пользовательским соглашением http://www . mathnet.ru/rus/agreement

Параметры загрузки:

IP : 54.224 .187 .69

26 апреля 2023 г., 16:45:29

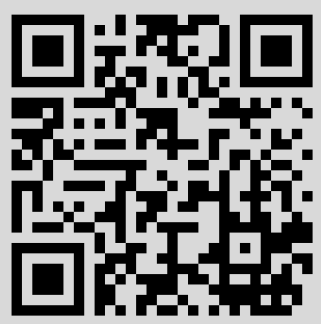




\title{
ФИЗИКА
}

Том 194, № 2

февраль, 2018

(C) 2018 г. $\quad$ Ф. Мухамедов* ${ }^{*}$ Ч. Паа ${ }^{\dagger}$, Х. Джамиль ${ }^{\dagger}$

\section{ОСНОВНЫЕ СОСТОЯНИЯ И ФАЗОВЫЕ ПЕРЕХОДЫ $\lambda$-МОДЕЛИ НА ДЕРЕВЕ КЭЛИ}

\begin{abstract}
Рассматривается $\lambda$-модель, которая является обобщением модели Поттса, со значениями спина $\{1,2,3\}$ на дереве Кэли порядка два. Для данной модели описаны основные состояния и доказано существование трансляционно-инвариантных мер Гиббса, что означает существование фазового перехода. Установлено существование 2-периодических мер Гиббса.
\end{abstract}

Ключевые слова: модель Поттса, основное состояние, мера Гиббса, фазовый переход.

DOI: https://doi.org/10.4213/tmf9309

\section{1. ВВЕДЕНИЕ}

Выбор гамильтониана для конкретных систем взаимодействующих частиц является важной задачей равновесной статистической механики [1]. Дело в том, что при рассмотрении реальной системы с большим (абстрактно говоря, бесконечным) числом степеней свободы невозможно учесть все свойства таких систем без исключений. Основная задача состоит в том, чтобы брать в расчёт только важнейшие свойства системы, а прочие осознанно не принимать во внимание. С другой стороны, главное назначение равновесной статистической механики - описание всех предельных распределений Гиббса, соответствующих заданному гамильтониану [2]. Эта задача может быть полностью решена только в относительно простых случаях. В частности, если в системе присутствуют только парные взаимодействия, описание предельных распределений Гиббса значительно упрощается.

Одними из важнейших моделей статистической механики являются модели Поттса. Они описывают особый класс систем статистической механики, который достаточно просто определить. Тем не менее их структура достаточно богата для описания практически любого мыслимого нюанса предмета исследования. В частности, эти модели оказались в центре недавнего всплеска интереса, вызванного соединением конформной теории поля, теории перколяции, теории узлов, квантовых групп

${ }^{*}$ Department of Mathematical Sciences, College of Science, The United Arab Emirates University, Al Ain, UAE. E-mail: far75m@gmail.com, farrukh.m@uaeu.ac.ae

${ }^{\dagger}$ Department of Computational and Theoretical Sciences, Faculty of Science, International Islamic University Malaysia, Kuantan, Malasia 
и интегрируемых систем [3], [4]. Заметим, что модель Поттса [5] была введена как обобщение модели Изинга для более чем двух компонент. В настоящее время модель Поттса охватывает ряд задач статистической физики (см., например, работу [6]).

Исследование фазовых переходов в спиновых моделях на иерархических решётках показало, что они могут помочь в точных вычислениях различных физических величин [7]-[10]. Изучение иерархических решёток началось с развития метода ренормгруппы Мигдала-Каданова, где они возникали как приближения обычных кристаллических решёток. В работах [11], [12] изучались фазовые диаграммы моделей Поттса с $q$ состояниями на решётках Бете и были найдены чистые фазы ферромагнитной модели Поттса. С использованием этих результатов в работах [13], [14] было построено несчётное число чистых фаз модели Поттса с тремя состояниями. Этот анализ был основан на разработанном в [11], [12], [15] подходе, опирающемся на теорию меры. Структура мер Гиббса модели Поттса изучалась в статьях [13], [16], [17].

Естественно рассмотреть усложнение модели Поттса, и в монографии [18] была предложена так называемая $\lambda$-модель на дереве Кэли (см. также [19], [20]). В данной модели учитываются разнообразные возможные взаимодействия (с ближайшими соседями). Для особого типа $\lambda$-моделей изучалась её неупорядоченная фаза [16], [21], а также исследовались некоторые алгебраические свойства таких моделей.

В настоящей работе мы рассматриваем симметричную $\lambda$-модель со значениями спина $\{1,2,3\}$ на дереве Кэли порядка два. Эта модель является более общей, чем модель Поттса, и обладает интересной структурой основных состояний. В разделе 3 (следующем после предварительного раздела 2) мы описываем основные состояния этой модели. В разделе 4 построены меры Гиббса. В разделе 5 доказано существование трансляционно-инвариантных мер Гиббса для $\lambda$-модели, что означает существование фазового перехода. Наконец, в разделе 6 установлено существование для данной модели 2-периодических мер Гиббса. Полученные в настоящей работе результаты значительно расширяют результаты работы [17], и, таким образом, открываются новые перспективы исследований моделей статистической механики на деревьях.

\section{2. ПРЕДВАРИТЕЛЬНЫЕ СВЕДЕНИЯ}

Пусть $\Gamma_{+}^{k}=(V, L)$ - полубесконечное дерево Кэли порядка $k \geqslant 1$ с корнем $x^{0}$ (из каждой вершины выходит ровно $k+1$ рёбер, кроме корня $x^{0}$, из которого выходит $k$ рёбер). Здесь $V$ - множество вершин, а $L$ - множество рёбер. Вершины $x$ и $y$ называются ближсайими соседями и обозначаются как $l=\langle x, y\rangle$, если существует соединяющее их ребро. Набор пар $\left\langle x, x_{1}\right\rangle, \ldots,\left\langle x_{d-1}, y\right\rangle$ называется путём из точки $x$ в точку $y$. Расстояние $d(x, y)$ между вершинами $x, y \in V$ на дереве Кэли равно длине кратчайшего пути из $x$ в $y$.

Введём обозначения

$$
W_{n}=\left\{x \in V: d\left(x, x^{0}\right)=n\right\}, \quad V_{n}=\bigcup_{m=1}^{n} W_{m}, \quad L_{n}=\left\{l=\langle x, y\rangle \in L: x, y \in V_{n}\right\} .
$$

Введём множество прямых потомков вершины $x$ :

$$
S(x)=\left\{y \in W_{n+1}: d(x, y)=1\right\}, \quad x \in W_{n} .
$$




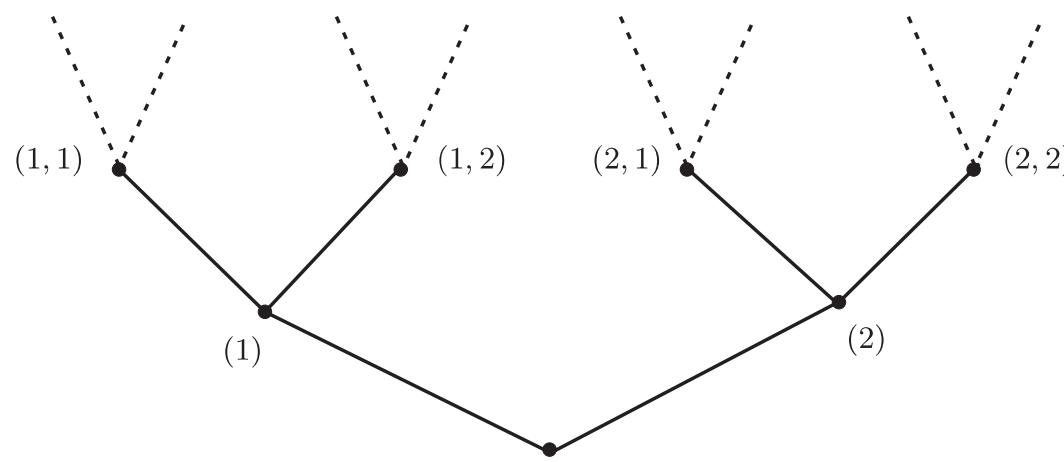

Уровень 3

(0)

Рис. 1. Первые уровни графа $\Gamma_{+}^{2}$.

Заметим, что любая вершина $x \neq x^{0}$ имеет $k$ прямых потомков, а корень $x^{0}$ имеет $k+1$ потомков.

Теперь введём координатную структуру на $\Gamma_{+}^{k}$. Каждая вершина $x$ (кроме $x^{0}$ ) графа $\Gamma_{+}^{k}$ имеет координаты $\left(i_{1}, \ldots, i_{n}\right) ;$ здесь $i_{m} \in\{1, \ldots, k\}, 1 \leqslant m \leqslant n$; вершине $x^{0}$ мы присвоим координату (0) (см. рис. 1). Другими словами, символ (0) обозначает уровень 0 , а $i_{1}, \ldots, i_{n}$ формируют уровень $n$ решётки. В этих обозначениях для $x=\left(i_{1}, \ldots, i_{n}\right)$ имеем $S(x)=\{(x, i): 1 \leqslant i \leqslant k\}$, где $(x, i)$ означает вершину с координатами $\left(i_{1}, \ldots, i_{n}, i\right)$.

Определим на $\Gamma_{+}^{k}$ бинарную операцию $\circ: \Gamma_{+}^{k} \times \Gamma_{+}^{k} \rightarrow \Gamma_{+}^{k}$ следующим образом: для любых двух элементов $x=\left(i_{1}, \ldots, i_{n}\right)$ и $y=\left(j_{1}, \ldots, j_{m}\right)$ положим

$$
\begin{aligned}
& x \circ y=\left(i_{1}, \ldots, i_{n}\right) \circ\left(j_{1}, \ldots, j_{m}\right)=\left(i_{1}, \ldots, i_{n}, j_{1}, \ldots, j_{m}\right) \\
& y \circ x=\left(j_{1}, \ldots, j_{m}\right) \circ\left(i_{1}, \ldots, i_{n}\right)=\left(j_{1}, \ldots, j_{m}, i_{1}, \ldots, i_{n}\right) .
\end{aligned}
$$

После введения операции $\Gamma_{+}^{k}$ становится некоммутативной полугруппой с единицей. Используя структуру полугруппы, определим сдвиги $\tau_{g}: \Gamma_{+}^{k} \rightarrow \Gamma_{+}^{k}$ для $g \in \Gamma_{k}$ как $\tau_{g}(x)=g \circ x$.

Пусть $G \subset \Gamma_{+}^{k}-$ подполугруппа в $\Gamma_{+}^{k}$, а $h: V \rightarrow \mathbb{R}-$ функция. Функция $h$ называется $G$-периодической, если $h\left(\tau_{g}(x)\right)=h(x)$ для всех $x \in V, g \in G$ и $l \in L$. Любая $\Gamma_{+}^{k}$-периодическая функция называется трансляиионно-инвариантной. Пусть

$$
G_{m}=\left\{x \in \Gamma_{+}^{k}: d\left(x, x^{0}\right) \equiv 0(\bmod m)\right\}, \quad m \geqslant 2 .
$$

Легко проверить, что $G_{m}$ является подполугруппой с единицей.

Приведём пример. Пусть $m=2, k=2$, тогда $G_{2}$ записывается следующим образом:

$$
G_{2}=\left\{(0),\left(i_{1}, i_{2}, \ldots, i_{2 n}\right), n \in \mathbb{N}\right\} .
$$

В данном случае $G_{2}$-периодическая функция $h$ имеет вид

$$
h(x)=\left\{\begin{array}{ll}
h_{1}, & x=\left(i_{1}, i_{2}, \ldots, i_{2 n}\right), \\
h_{2}, & x=\left(i_{1}, i_{2}, \ldots, i_{2 n+1}\right),
\end{array} \quad i_{k} \in\{1,2\}, \quad k \in V .\right.
$$


В настоящей работе мы рассматриваем модели, в которых спин принимает значения из множества $\Phi=\{1,2, \ldots, q\}$ и приписан к вершинам дерева. Конфигурация $\sigma$ на $V$ определяется как функция $x \in V \rightarrow \sigma(x) \in \Phi$; множество всех конфигураций совпадает с $\Omega=\Phi^{\Gamma^{k}}$. Гамильтониан $\lambda$-модели имеет следующий вид:

$$
H(\sigma)=\sum_{\langle x, y\rangle \in L} \lambda(\sigma(x), \sigma(y))
$$

где сумма берётся по всем парам ближайших соседей $\langle x, y\rangle$ и $\sigma \in \Omega$. С физической точки зрения взаимодействие между частицами не зависит от их положения, поэтому далее мы предполагаем, что $\lambda$ - симметричная функция, т. е. $\lambda(u, v)=\lambda(v, u)$ для всех $u, v \in \mathbb{R}$. Заметим, что $\lambda$-модель данного типа можно рассматривать как обобщение модели Поттса, которая соответствует $\lambda(x, y)=-J \delta_{x y}$, где $x, y, J \in \mathbb{R}$. В дальнейшем мы ограничиваемся случаем $k=2$ и $\Phi=\{1,2,3\}$ и для простоты рассматриваем функцию

$$
\lambda(i, j)= \begin{cases}\bar{a}, & |i-j|=2, \\ \bar{b}, & |i-j|=1 \\ \bar{c}, & i=j,\end{cases}
$$

где $\bar{a}, \bar{b}, \bar{c}$ - некоторые заданные действительные числа.

ЗАмечание 1. Рассматриваемая модель является более общей, чем известная модель Поттса [6], в модель Поттса она переходит при $\bar{a}=\bar{b}=0, \bar{c} \neq 0$.

\section{3. ОСНОВНЫЕ СОСТОЯНИЯ}

В данном разделе мы описываем основное состояние $\lambda$-модели на дереве Кэли. Для пары конфигураций $\sigma$ и $\varphi$, совпадающих почти всюду, т. е. везде, кроме конечного числа точек, рассмотрим относительный гамильтониан $H(\sigma, \varphi)$, определяющий разность энергий конфигураций $\sigma$ и $\varphi$ :

$$
H(\sigma, \varphi)=\sum_{\langle x, y\rangle \in L}(\lambda(\sigma(x), \sigma(y))-\lambda(\varphi(x), \varphi(y))) .
$$

Для каждого $x \in V$ множество $\{x, S(x)\}$ называется шаром и обозначается как $b_{x}$ или просто $b$. Множество всех шаров обозначим через $M$. Определим энергию конфигурации $\sigma_{b}$ на шаре $b$ следующим образом:

$$
U\left(\sigma_{b}\right)=\frac{1}{2} \sum_{\langle x, y\rangle \in L} \lambda(\sigma(x), \sigma(y))
$$

Из (4) получаем следующую лемму.

ЛЕмма 1. Относителъный гамильтониан (4) имеет вид

$$
H(\sigma, \varphi)=\sum_{b \in M}\left(U\left(\sigma_{b}\right)-U\left(\varphi_{b}\right)\right)
$$

Также справедливо следующее утверждение. 


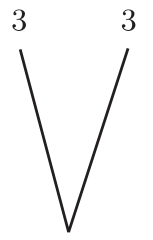

1

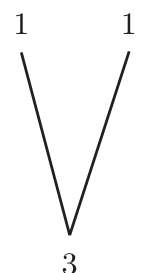

3

Рис. 2. Конфигурации для множества $A_{1}$.

ЛЕмма 2. Для любой конфигурации $\varphi_{b}$ на $b($ әд $b \in M)$ имеет место включение

$$
U\left(\varphi_{b}\right) \in\left\{\frac{\alpha+\beta}{2}: \alpha, \beta \in\{\bar{a}, \bar{b}, \bar{c}\}\right\}
$$

Конфигурация $\varphi$ называется основным состоянием относительного гамильтониана $H$, если для любого $b \in M$

$$
U\left(\varphi_{b}\right)=\min \left\{\frac{\alpha+\beta}{2}: \alpha, \beta \in\{\bar{a}, \bar{b}, \bar{c}\}\right\} .
$$

Для любой конфигурации $\sigma_{b}$, имеем $U\left(\sigma_{b}\right) \in\left\{U_{1}, U_{2}, U_{3}, U_{4}, U_{5}, U_{6}\right\}$, где

$$
U_{1}=\bar{a}, \quad U_{2}=\frac{\bar{a}+\bar{b}}{2}, \quad U_{3}=\frac{\bar{a}+\bar{c}}{2}, \quad U_{4}=\bar{b}, \quad U_{5}=\frac{\bar{b}+\bar{c}}{2}, \quad U_{6}=\bar{c} .
$$

Положим

$$
A_{m}=\left\{(\bar{a}, \bar{b}, \bar{c}) \in \mathbb{R}^{3}: U_{m}=\min _{1 \leqslant k \leqslant 6} U_{k}\right\}
$$

Получаем

$$
\begin{array}{ll}
A_{1}=\left\{(\bar{a}, \bar{b}, \bar{c}) \in \mathbb{R}^{3}: \bar{a} \leqslant \bar{b}, \bar{a} \leqslant \bar{c}\right\}, & A_{2}=\left\{(\bar{a}, \bar{b}, \bar{c}) \in \mathbb{R}^{3}: \bar{a}=\bar{b} \leqslant \bar{c}\right\}, \\
A_{3}=\left\{(\bar{a}, \bar{b}, \bar{c}) \in \mathbb{R}^{3}: \bar{a}=\bar{c} \leqslant \bar{b}\right\}, & A_{4}=\left\{(\bar{a}, \bar{b}, \bar{c}) \in \mathbb{R}^{3}: \bar{b} \leqslant \bar{a}, \bar{b} \leqslant \bar{c}\right\}, \\
A_{5}=\left\{(\bar{a}, \bar{b}, \bar{c}) \in \mathbb{R}^{3}: \bar{b}=\bar{c} \leqslant \bar{a}\right\}, & A_{6}=\left\{(\bar{a}, \bar{b}, \bar{c}) \in \mathbb{R}^{3}: \bar{c} \leqslant \bar{a}, \bar{c} \leqslant \bar{b}\right\} .
\end{array}
$$

Теперь найдём основные состояния для каждого представленного случая. Введём некоторые новые обозначения. Для каждой последовательности $\left\{k_{0}, k_{1}, \ldots, k_{n}, \ldots\right\}$, где $k_{n} \in\{1,2,3\}, n \in \mathbb{N} \cup\{0\}$, определим конфигурацию $\sigma$ на $\Omega$ как $\sigma(x)=k_{\ell}$, если $x \in W_{\ell}, \ell \geqslant 0$. Обозначим эту конфигурацию через $\sigma_{\left[k_{n}\right]}$. Если последовательность $\left\{k_{0}, k_{1}, \ldots, k_{n}, \ldots\right\}$ является $n$-периодической (т. е. $k_{\ell+n}=k_{\ell}$ для всех $n \in \mathbb{N}$ ), то вместо $\left\{k_{0}, k_{1}, \ldots, k_{n}, \ldots\right\}$ мы пишем $\left\{k_{0}, k_{1}, \ldots, k_{n-1}\right\}$. Соответствующая конфигурация обозначается как $\sigma_{\left[k_{0}, k_{1}, \ldots, k_{n-1}\right]}$.

Теорема 1. Пусть $(\bar{a}, \bar{b}, \bar{c}) \in A_{1}$, тогда существуют только два $G_{2}$-периодических основных состояния.

ДокАЗАтЕЛьСтво. Пусть $(\bar{a}, \bar{b}, \bar{c}) \in A_{1}$, тогда минимальное значение из этих трёх чисел - это $\bar{a}$, при этом минимум достигается на конфигурации на шаре $b$, показанной на рис. 2. 

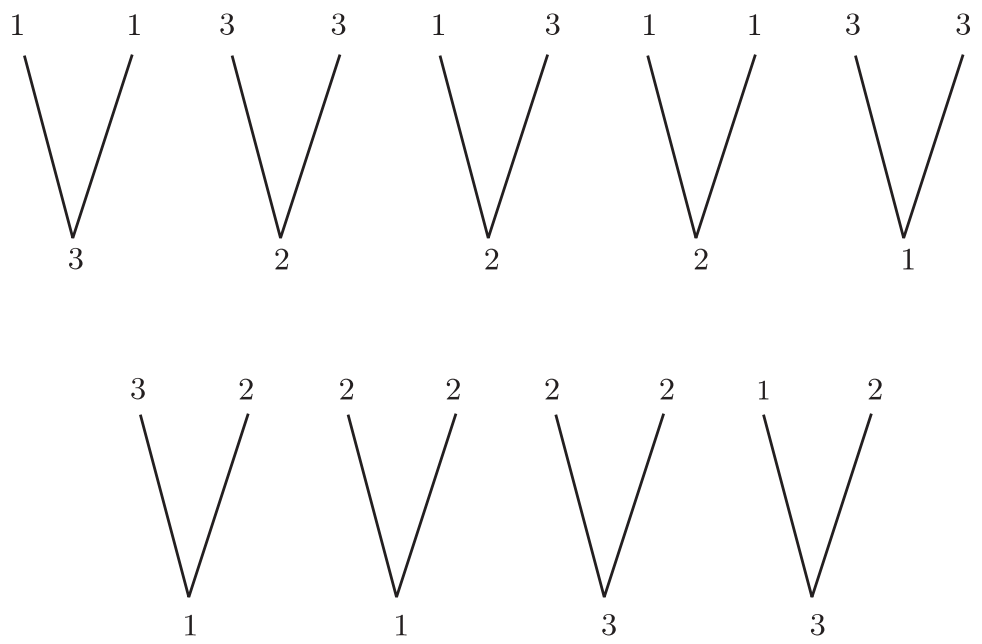

Рис. 3. Конфигурации для множества $A_{2}$.

Теперь, используя рис. 2 , для любого $n \in \mathbb{N}$ можно построить конфигурации на $\Omega$ вида $\sigma_{1}^{(2)}=\sigma_{[1,3]}, \sigma_{2}^{(2)}=\sigma_{[3,1]}$. Тогда для любого $b \in M$ мы имеем

$$
U\left(\sigma_{b_{1,2}}^{(2)}\right)=\min _{1 \leqslant k \leqslant 6} U_{k}
$$

следовательно, $\sigma_{1}^{(2)}$ и $\sigma_{2}^{(2)}$ - основные состояния. Кроме того, конфигурации $\sigma_{1}^{(2)}$ и $\sigma_{2}^{(2)}$ являются $G_{2}$-периодическими. Заметим, что все основные состояния совпадают с указанными выше.

ТеОРема 2. Пусть $(\bar{a}, \bar{b}, \bar{c}) \in A_{2}$, тогда верны следующие утверждения:

1) для любого $n \in \mathbb{N}$ существует $G_{n}$-периодическое основное состояние;

2) существует несчётное число основных состояний.

ДокАЗАтЕЛьство. Пусть $(\bar{a}, \bar{b}, \bar{c}) \in A_{2}$, тогда минимальное значение из этих трёх чисел - это $(\bar{a}+\bar{b}) / 2$, при этом минимум достигается на конфигурации на шаре $b$, показанной на рис. 3 .

1. Используя рис. 3 , для любого $n \in \mathbb{N}$ можно построить конфигурации на $\Omega$ вида

$$
\sigma^{(2 n)}=\sigma_{2 n}^{[1,(2,3), \ldots,(2,3), 2]}, \quad \sigma^{(2 n+1)}=\sigma^{[1,(2,3), \ldots,(2,3)]} .
$$

Тогда для любого $b \in M$ мы имеем

$$
U\left(\sigma^{(\xi)}\right)=\min _{1 \leqslant k \leqslant 6} U_{k}, \quad \xi \in\{2 n, 2 n+1\},
$$

следовательно, $\sigma^{(n)}$ есть $G_{n}$-периодическое основное состояние.

2. Для построения несчётного множества основных состояний рассмотрим множество

$$
\Sigma_{1,2,3}=\left\{\left(\mathbf{t}_{n}\right): \mathbf{t}_{n} \in\{1,2,3\}, \delta_{\left\{t_{n}, t_{n+1}\right\}}=0, n \in \mathbb{N}\right\}
$$



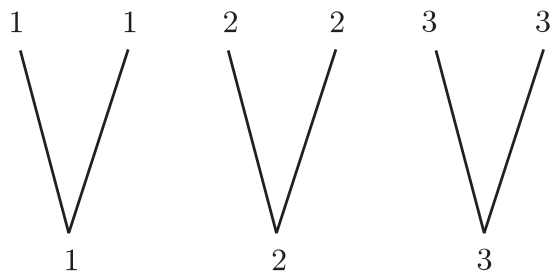

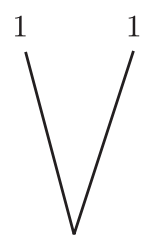

3

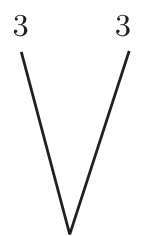

1

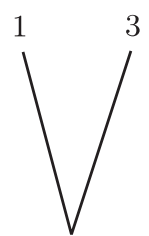

1

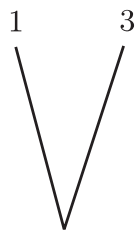

3

Рис. 4. Конфигурации для множества $A_{3}$.

где $\delta$ - символ Кронекера. Легко убедиться, что множество $\Sigma_{1,2,3}$ несчётно. Возьмём любое $\mathbf{t}=\left(t_{n}\right) \in \Sigma_{1,2,3}$. Построим конфигурацию следующим образом:

$$
\sigma^{(\mathbf{t})}= \begin{cases}1, & x=(0), \\ \mathbf{t}_{k}, & x \in W_{k}, k \in V .\end{cases}
$$

Нетрудно проверить, что $\sigma^{(\mathbf{t})}$ является основным состоянием, при этом соответствие $\mathbf{t} \in \Sigma_{1,2,3} \rightarrow \sigma^{(\mathbf{t})}$ показывает, что множество $\left\{\sigma^{(\mathbf{t})}: \mathbf{t} \in \Sigma_{1,2,3}\right\}$ несчётно. Доказательство завершено.

ТеОрема 3. Пусть $(\bar{a}, \bar{b}, \bar{c}) \in A_{3}$, тогда верны следующие утвержсдения:

1) существуют три трансляционно-инвариантных основных состояния;

2) для любого $n \in \mathbb{N}$ существует $G_{n}$-периодическое основное состояние.

ДоказАтельство. Пусть $(\bar{a}, \bar{b}, \bar{c}) \in A_{3}$, тогда минимальное значение из этих трёх чисел - это $(\bar{a}+\bar{c}) / 2$, при этом минимум достигается на конфигурации на шаре $b$, показанной на рис. 4.

1. В данном случае, мы имеем три конфигурации, которые являются трансляционно-инвариантными основными состояниями: $\sigma^{(k)}=\sigma_{[k]}, k=\{1,2,3\}$.

2. Используя рис. 4 , для любого $n \in \mathbb{N}$ можно построить следующую конфигурацию на $\Omega: \sigma^{(n)}=\sigma_{n}^{[1,3, \ldots, 3]}$. Тогда для любого $b \in M$ мы имеем

$$
U\left(\sigma^{(n)}\right)=\min _{1 \leqslant k \leqslant 6} U_{k}
$$

следовательно, $\sigma^{(n)}$ является $G_{n}$-периодическим основным состоянием.

Теорема 4. Пусть $(\bar{a}, \bar{b}, \bar{c}) \in A_{4}$, тогда для любого $n \in \mathbb{N}$ существует $G_{(3 n+1)}$ периодическое основное состояние. 

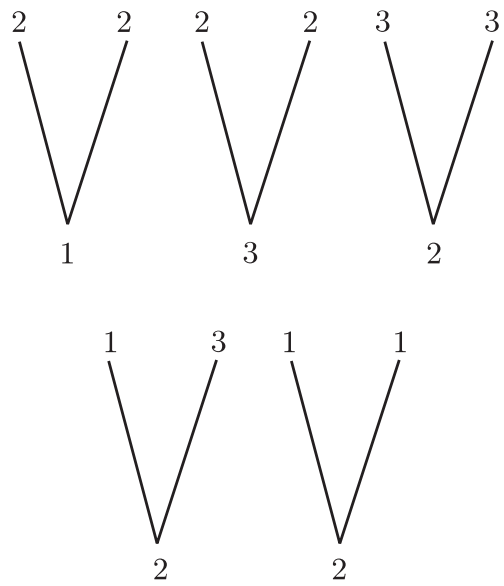

Рис. 5. Конфигурации для множества $A_{4}$.

ДокАЗАТЕльСТво. Пусть $(\bar{a}, \bar{b}, \bar{c}) \in A_{3}$, тогда минимальное значение из этих трёх чисел - это $\bar{b}$, при этом минимум достигается на конфигурации на шаре $b$, показанной на рис. 5 .

Используя рис. 5 , для любого $n \in \mathbb{N}$ можно построить следующую конфигурацию на $\Omega: \sigma^{(3 n+1)}=\sigma_{[1,(\underbrace{(2,3,2), \ldots,(2,3,2)}_{n}]}$. Тогда для любого $b \in M$ мы имеем

$$
U\left(\sigma^{(3 n+1)}\right)=\min _{1 \leqslant k \leqslant 6} U_{k},
$$

следовательно, $\sigma^{(3 n+1)}$ является $G_{(3 n+1)}$-периодическим основным состоянием.

Теорема 5. Пусть $(\bar{a}, \bar{b}, \bar{c}) \in A_{5}$, тогда вернъ следующие утверждения:

1) существуют три трансляиионно-инвариантных основных состояния;

2) для любого $n \in \mathbb{N}$ существует $G_{n}$-периодическое основное состояние;

3) существует несчётное количество основных состояний.

ДокАЗАТЕльСТво. Пусть $(\bar{a}, \bar{b}, \bar{c}) \in A_{5}$, тогда минимальное значение из этих трёх чисел - это $(\bar{b}+\bar{c}) / 2$, при этом минимум достигается на конфигурации на шаре $b$, показанной на рис. 6 .

1. В данном случае существуют три конфигурации, которые являются трансляционно-инвариантными основными состояниями: $\sigma^{(k)}=\sigma_{[k]}, k=\{1,2,3\}$.

2. Используя рис. 6 , для любого $n \in \mathbb{N}$ можно построить следующую конфигурацию на $\Omega: \sigma^{(n)}=\sigma_{[1,2, \ldots, 2]}$. Тогда для любого $b \in M$ имеем

$$
U\left(\sigma^{(n)}\right)=\min _{1 \leqslant k \leqslant 6} U_{k},
$$

следовательно, $\sigma^{(n)}$ является $G_{n}$-периодическим основным состоянием.

3. Для построения несчётного множества основных состояний рассмотрим множество

$$
\Sigma_{2,3}=\left\{\left(\mathbf{t}_{n}\right): \mathbf{t}_{n} \in\{2,3\}, n \in \mathbb{N}\right\},
$$




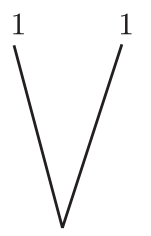

1

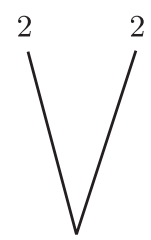

3

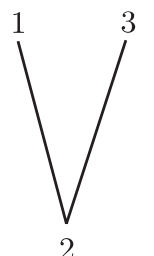

2

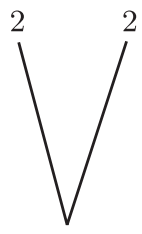

2

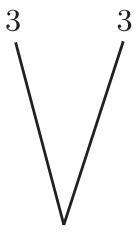

3

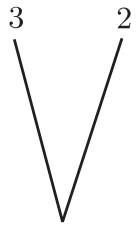

3

Рис. 6. Конфигурации для множества $A_{5}$.
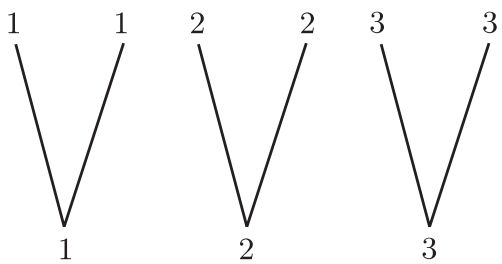

Рис. 7. Конфигурации для множества $A_{6}$.

которое является несчётным. Выберем произвольно $\mathbf{t}=\left(t_{n}\right) \in \Sigma_{2,3}$. Построим конфигурацию следующим образом:

$$
\sigma^{(\mathbf{t})}= \begin{cases}2, & x=(0), \\ \mathbf{t}_{k}, & x \in W_{k}, k \in V .\end{cases}
$$

Несложно проверить, что $\sigma^{(\mathbf{t})}$ является основным состоянием, при этом соответствие $\mathbf{t} \in \Sigma_{2,3} \rightarrow \sigma^{(t)}$ показывает, что множество $\left\{\sigma^{(\mathbf{t})}, \mathbf{t} \in \Sigma_{2,3}\right\}$ несчётно.

Теорема 6. Пусть $(\bar{a}, \bar{b}, \bar{c}) \in A_{6}$, тогда существуют только три трансляционно-инвариантных основных состояния.

ДокАзАтЕЛЬство. Пусть $(\bar{a}, \bar{b}, \bar{c}) \in A_{5}$, тогда минимальное значение из этих трёх чисел - это $\bar{c}$, при этом минимум достигается на конфигурации на шаре $b$, показанной на рис. 7. В этом случае мы имеем три конфигурации $\sigma^{(k)}=\sigma_{[k]}, k=\{1,2,3\}$, которые являются трансляционно-инвариантными основными состояниями. 


\section{4. ПОСТРОЕНИЕ ГИББСОВСКИХ СОСТОЯНИЙ ДЛЯ $\lambda$-МОДЕЛИ}

Определим конечномерное распределение вероятностной меры $\mu^{(n)}$ в объёме $V_{n}$ :

$$
\mu^{(n)}\left(\sigma_{n}\right)=\frac{1}{Z_{n}} \exp \left\{\beta H_{n}\left(\sigma_{n}\right)+\sum_{x \in W_{n}} h_{\sigma(x), x}\right\}, \quad \sigma_{n} \in \Phi^{V_{n}},
$$

где $\beta=1 / T, T>0$ - температура и

$$
Z_{n}=\sum_{\sigma \in \Phi^{V_{n}}} \exp \left\{\beta H_{n}\left(\sigma_{n}\right)+\sum_{x \in W_{n}} h_{\sigma(x), x}\right\}
$$

есть нормировочный множитель. Здесь $\left\{h_{x}=\left(h_{1, x}, \ldots, h_{q, x}\right) \in \mathbb{R}^{q}, x \in V\right\}$ - множество векторов и

$$
H_{n}\left(\sigma_{n}\right)=\sum_{\langle x, y\rangle \in L_{n}} \lambda(\sigma(x), \sigma(y))
$$

Последовательность вероятностных распределений $\left\{\mu^{(n)}\right\}$ называется согласованной, если для всех $n \geqslant 1$ и $\sigma_{n-1} \in \Phi^{V_{n-1}}$

$$
\sum_{\omega_{n} \in \Phi^{W_{n}}} \mu^{(n)}\left(\sigma_{n-1} \vee \omega_{n}\right)=\mu^{(n-1)}\left(\sigma_{n-1}\right),
$$

где через $\sigma_{n-1} \vee \omega_{n}$ обозначено объединение всех конфигураций. В данном случае мы имеем единственную меру $\mu$ на $\Phi^{V}$ такую, что для всех $n$ и $\sigma_{n} \in \Phi^{V_{n}}$

$$
\mu\left(\left\{\sigma \mid V_{n}=\sigma_{n}\right\}\right)=\mu^{(n)}\left(\sigma_{n}\right),
$$

которая называется расщеплённой мерой Гиббса, соответствующей гамильтониану (2) и векторнозначной функции $h_{x}, x \in V$ (о расщеплённых мерах см. в монографии [20]).

Следующее утверждение описывает условие на $h_{x}$, которое обеспечивает согласованность семейства мер $\left\{\mu^{(n)}\right\}$.

Теорема 7. Меры $\mu^{(n)}, n=1,2, \ldots$, удовлетворяют условию согласования тогда и только тогда, когда для любого $x \in V$ выполнены следующие уравнения:

$$
u_{k, x}=\prod_{y \in S(x)} \frac{\sum_{j=1}^{q-1} e^{\beta \lambda(k, j)} u_{j, y}+e^{\beta \lambda(k, q)}}{\sum_{j=1}^{q-1} e^{\beta \lambda(q, j)} u_{j, y}+e^{\beta \lambda(q, q)}}, \quad k=1, \ldots, q-1,
$$

где $u_{k, x}=e^{h_{k, x}-h_{q, x}}$.

ДоказАтельство. Докажем необходимость (13). Согласно условию согласования (12)

$$
\begin{aligned}
\sum_{w \in \Phi V_{n}} \frac{1}{Z_{n}} & \exp \left\{\beta H_{n}\left(\sigma_{n-1} \vee \omega\right)+\sum_{x \in W_{n}} h_{\omega(x), x}\right\}= \\
& \left.=\frac{1}{Z_{n-1}} \exp \left\{\beta H_{n-1}\left(\sigma_{n-1}\right)+\sum_{x \in W_{n-1}} h_{\sigma(x), x}\right)\right\} .
\end{aligned}
$$


Принимая во внимание, что

$$
\begin{aligned}
H_{n}\left(\sigma_{n-1} \vee \omega_{n}\right) & =\sum_{\sigma \in \Phi^{V_{n}-1}} \lambda(\sigma(x), \sigma(y))+\sum_{\substack{x \in W_{n-1}, y \in S(x)}} \lambda(\sigma(x), \omega(y))= \\
& =H_{n-1}(\sigma)+\sum_{x \in W_{n-1}} \sum_{y \in S(x)} \lambda(\sigma(x), \omega(y)),
\end{aligned}
$$

имеем

$$
\begin{aligned}
\frac{Z_{n-1}}{Z_{n}} \sum_{\omega \in \Phi^{V_{n}}} & \exp \left\{\beta \sum_{x \in W_{n-1}} \sum_{y \in S(x)} \lambda(\sigma(x), \omega(y))+\beta \sum_{x \in W_{n-1}} \sum_{y \in S(x)} h_{\omega(x),(x)}\right\}= \\
& =\exp \left\{\sum_{x \in W_{n-1}} h_{\sigma(x),(x)}\right\},
\end{aligned}
$$

откуда следует, что

$$
\frac{Z_{n-1}}{Z_{n}} \prod_{x \in W_{n-1}} \prod_{y \in S(x)} \sum_{\omega \in \Phi^{V_{n}}} e^{\beta \lambda(\sigma(x), \omega(y))+h_{\omega(y),(y)}}=\prod_{x \in W_{n-1}} e^{h_{\sigma(x),(x)}} .
$$

Рассматривая такие конфигурации $\bar{\sigma}^{(k)} \in \Phi^{v_{n-1}}$ для $\Phi=\{1, \ldots, q\}$, что $\sigma(x)=k$ при фиксированном $x \in V$ и $k=1, \ldots, q$, и деля уравнение $(14)$ при $\sigma(x)=\bar{\sigma}^{(k)}$ на уравнение (14) при $\sigma(x)=\bar{\sigma}^{(q)}$, получаем

$$
\prod_{y \in S(x)} \frac{\sum_{w \in \Phi} e^{\beta \lambda(k, \omega(y))+h_{\omega(y), y}}}{\sum_{\omega \in \Phi} e^{\beta \lambda(q, \omega(y))+h_{\omega(y), y}}}=\frac{e^{h_{k, x}}}{e^{h_{q, x}}} .
$$

Таким образом,

$$
\prod_{y \in S(x)} \frac{\sum_{w \in \Phi}^{q} e^{\beta \lambda(k, j)+h_{j, y}}}{\sum_{w \in \Phi}^{q} e^{\beta \lambda(q, j)+h_{j, y}}}=e^{h_{k, x}-h_{q, x}}
$$

Отсюда, положив $u_{k, x}=e^{h_{k, x}-h_{q, x}}$, выводим, что

$$
\prod_{y \in S(x)} \frac{\sum_{j=1}^{q-1} e^{\beta \lambda(k, j)+u_{j, y}}+e^{\beta \lambda(k, q)}}{\sum_{j=1}^{q-1} e^{\beta \lambda(q, j)+u_{j, y}}+e^{\beta \lambda(q, q)}}=u_{k, x} .
$$

Теперь докажем достаточность. Предположим, что уравнение (12) выполняется, тогда получаем (16). Это даёт

$$
\prod_{y \in S(x)} \sum_{j=1}^{q} e^{\beta \lambda(k, j)+h_{j, y}}=a(x) e^{h_{k, x}}, \quad k=1, \ldots, q, \quad x \in W_{n-1},
$$

для некоторой положительной функции $a(x), x \in V$. Вычислим произведение равенств (18) по $x \in W_{n-1}$ и получим, что для любой конфигурации $\sigma \in \Phi_{n-1}^{V}$

$$
\prod_{x \in W_{n-1}} \prod_{y \in S(x)} \sum_{j=1}^{q} e^{\beta \lambda(\sigma(x), j)+h_{j, y}}=\prod_{x \in W_{n-1}} a(x) e^{h_{k, x}}
$$


Пусть $A_{n-1}=\prod_{x \in W_{n-1}} a(x)$, тогда из (19) имеем

$$
\prod_{x \in W_{n-1}} \prod_{y \in S(x)} \sum_{w \in \Phi} e^{\beta \lambda(\sigma(x), \omega(y))+h_{\omega, y}}=A_{n} \prod_{x \in W_{n-1}} e^{h_{\sigma(x), x}} .
$$

Умножим обе части этого равенства на $e^{\beta H_{n-1}(\sigma)}$ и получим

$$
e^{\beta H_{n-1}(\sigma)} \prod_{x \in W_{n-1}} \prod_{y \in S(x)} \sum_{w \in \Phi} e^{\beta \lambda(\sigma(x), \omega(y))+h_{\omega, y}}=A_{n} e^{\beta H_{n-1}(\sigma)} \prod_{x \in W_{n-1}} e^{h_{\sigma(x), x}},
$$

что даёт

$$
Z_{n} \sum_{\omega_{n} \in \Phi^{V_{n}}} \mu^{(n)}\left(\sigma \vee \omega_{n}\right)=A_{n-1} Z_{n-1} \mu^{(n-1)}(\sigma)
$$

Поскольку $\mu^{(n)}\left(\sigma \vee \omega_{n}\right)$ для любого $n \geqslant 1$ есть вероятностная мера, мы имеем

$$
\sum_{\omega_{n} \in \Phi^{V_{n}}} \mu^{(n)}\left(\sigma \vee \omega_{n}\right)=\mu^{(n-1)}(\sigma)=1
$$

и из (21) получаем, что $Z_{n}=A_{n-1} Z_{n-1}$. Доказательство завершено.

\section{5. ОПИСАНИЕ ТРАНСЛЯЦИОННО-ИНВАРИАНТНЫХ МЕР ГИББСА}

В настоящем разделе установлено существование фазового перехода для $\lambda$-модели, заданной в (3). Как и прежде, предполагаем, что $k=2, q=3$.

Чтобы установить наличие фазового перехода, найдём трансляционно-инвариантные меры Гиббса. Здесь трансляционно-инвариантная мера Гиббса понимается как расщеплённая мера Гиббса, отвечающая решению $\mathbf{u}_{x}$ уравнения (17). Данное решение трансляционно-инвариантно, т. е. $\mathbf{u}_{x}=\mathbf{u}_{y}$ для всех $x, y \in V$. Это означает, что $\mathbf{u}_{x}=\mathbf{u}$, где $\mathbf{u}=\left(u_{1}, u_{2}\right), u_{1}, u_{2}>0$. В силу теоремы 7 величины $u_{1}$ и $u_{2}$ должны удовлетворять следующим уравнениям:

$$
u_{1}=\left(\frac{\theta_{1} u_{1}+\theta_{2} u_{2}+\theta_{3}}{\theta_{3} u_{1}+\theta_{2} u_{2}+\theta_{1}}\right)^{2}, \quad u_{2}=\left(\frac{\theta_{2} u_{1}+\theta_{1} u_{2}+\theta_{2}}{\theta_{3} u_{1}+\theta_{2} u_{2}+\theta_{1}}\right)^{2},
$$

где $\theta_{1}=e^{\beta \bar{c}}, \theta_{2}=e^{\beta \bar{b}}, \theta_{3}=e^{\beta \bar{a}}$ (здесь использовано соотношение $\left.(3)\right)$.

Из (22) можно увидеть, что прямая $u_{1}=1$ инвариантна относительно этих уравнений. Таким образом, уравнение на этой инвариантной линии сводится к

$$
u_{2}=\left(\frac{\theta_{1} u_{2}+2 \theta_{2}}{\theta_{2} u_{2}+\theta_{1}+\theta_{3}}\right)^{2} .
$$

Положим

$$
x=\frac{u_{2} \theta_{1}}{2 \theta_{2}}, \quad a=\frac{1}{8 \theta_{2}^{5}}, \quad b=\frac{\theta_{1}\left(\theta_{1}+\theta_{3}\right)}{2 \theta_{2}^{2}}
$$

и перепишем уравнение $(23)$ как

$$
a x=\left(\frac{1+x}{b+x}\right)^{2} .
$$

Так как $x>0, k \geqslant 1, a>0$ и $b>0$, из предложения 10.7 монографии [15] вытекает следующее утверждение. 
ЛЕмма 3. Множество решений уравнения (25) устроено следующим образом.

1. Если $b \leqslant 9$, то решение уравнения (25) единственно.

2. Если $b>9$, то существуют $\eta_{1}=\eta_{1}(b)$ u $\eta_{2}=\eta_{2}(b)$ такие, что $0<\eta_{1}<\eta_{2}$, и тогда при $\eta_{1}<a<\eta_{2}$ уравнение (25) имеет три решения.

3. Если $a=\eta_{1}$ или $a=\eta_{2}$, где величины $\eta_{1}$ и $\eta_{2}$ определяются как

$$
\eta_{i}(b)=\frac{1}{x_{i}}\left(\frac{1+x_{i}}{b+x_{i}}\right)^{2}, \quad i=1,2,
$$

a $x_{1}$ и $x_{2}-$ корни уравнения $x^{2}+(3-b) x+b=0$, то уравнение $(25)$ имеет два решения.

Из этой леммы получаем следующий результат.

ТЕОРема 8. Если условие 2 леммы 3 выполняется, то происходит фазовый переход.

Рассмотрим конкретный пример. Пусть $b=10$ и, следовательно, $x_{1}=2, x_{2}=5$. Тогда $\eta_{1}=1 / 32$ и $\eta_{2}=4 / 125$. По теореме 8 заключаем, что если

$$
\frac{1}{32}<\frac{2 \theta_{2}^{3}}{\theta_{1}^{3}}<\frac{4}{125}
$$

то происходит фазовый переход.

\section{6. ПЕРИОДИЧЕСКАЯ МЕРА ГИББСА}

В данном разделе изучаются 2-периодические меры Гиббса. Напомним, что функция $\mathbf{u}_{x}$ является 2-периодической, если $\mathbf{u}_{x}=\mathbf{u}_{y}$, когда $d(x, y)$ делится на 2 (детали см. в разделе 2).

Пусть $\mathbf{u}_{x}$ есть 2-периодическая функция. Чтобы существовала соответствующая мера Гиббса, функция $\mathbf{u}_{x}=\left(u_{x, 1}, u_{x, 2}\right)$ должна удовлетворять следующим уравнениям:

$$
u_{x, 1}=\left(\frac{\theta_{1} u_{y, 1}+\theta_{2} u_{y, 2}+\theta_{3}}{\theta_{3} u_{y, 1}+\theta_{2} u_{y, 2}+\theta_{1}}\right)^{2}, \quad u_{x, 2}=\left(\frac{\theta_{2} u_{y, 1}+\theta_{1} u_{y, 2}+\theta_{2}}{\theta_{3} u_{y, 1}+\theta_{2} u_{y, 2}+\theta_{1}}\right)^{2},
$$

где $d(x, y)=2$ для всех $x, y \in V$.

Согласно результатам предыдущего раздела $u_{x, 1}=1$ является инвариантной прямой для уравнения (27). Таким образом, в дальнейшем будем предполагать, что $u_{x, 1}=1$ для всех $x \in V$. Тогда уравнение (27) сводится к

$$
u=f(f(u)), \quad f(u)=\left(\frac{\theta_{1} u+2 \theta_{2}}{\theta_{2} u+\theta_{1}+\theta_{3}}\right)^{2} .
$$

Корни уравнения $u_{2}=f(u)$, очевидно, являются корнями уравнения (28). Для нахождения других корней уравнения (28) необходимо рассмотреть уравнение

$$
\frac{f(f(u))-u}{f(u)-u}=0
$$


которое даёт квадратное уравнение

$$
\begin{aligned}
& \left(\theta_{2}^{2} \theta_{3}^{2}+2 \theta_{2} \theta_{1}^{2} \theta_{3}+2 \theta_{3} \theta_{2}^{2} \theta_{1}+\theta_{1}^{4}+2 \theta_{2} \theta_{1}^{3}+\theta_{1}^{2} \theta_{2}^{2}\right) u^{2}+ \\
& +\left(\theta_{1}^{2} \theta_{3}^{2}+6 \theta_{2} \theta_{1}^{3}+8 \theta_{1}^{2} \theta_{2}^{2}+8 \theta_{3} \theta_{2}^{2} \theta_{1}+2 \theta_{2} \theta_{3}^{3}+2 \theta_{1}^{3} \theta_{2}+6 \theta_{2} \theta_{1}^{2} \theta_{3}-4 \theta_{2}^{4}+6 \theta_{2} \theta_{3}^{2} \theta_{1}+\theta_{1}^{4}\right) u+ \\
& +\theta_{1}^{4}+4 \theta_{1}^{2} \theta_{2}^{2}+4 \theta_{2} \theta_{1}^{3}+\theta_{3}^{4}+4 \theta_{1}^{3} \theta_{3}+4 \theta_{2} \theta_{3}^{2} \theta_{1}+6 \theta_{1}^{2} \theta_{3}^{2}+8 \theta_{2} \theta_{1}^{2} \theta_{3}+4 \theta_{1} \theta_{3}^{3}=0 .
\end{aligned}
$$

Заметим, что положительные корни этого уравнения задают периодические меры Гиббса. В общем случае существование двух положительных корней задаётся условиями $B<0, D>0$, где $B$ - коэффициент при линейном по $u$ члене в $(29)$,

$$
\begin{aligned}
D=16 & \theta_{2}^{8}-3 \theta_{1}^{8}+96 \theta_{2}^{3} \theta_{1}^{4} \theta_{3}-64 \theta_{3} \theta_{2}^{6} \theta_{1}+40 \theta_{1}^{2} \theta_{3}^{2} \theta_{2}^{4}-40 \theta_{1}^{4} \theta_{3}^{3} \theta_{2}-48 \theta_{2}^{5} \theta_{1}^{2} \theta_{3}- \\
& -48 \theta_{2}^{5} \theta_{3}^{2} \theta_{1}-20 \theta_{1}^{3} \theta_{3}^{4} \theta_{2}-48 \theta_{1}^{5} \theta_{3}^{2} \theta_{2}+64 \theta_{1}^{2} \theta_{2}^{3} \theta_{3}^{3}-4 \theta_{1}^{2} \theta_{3}^{5} \theta_{2}+16 \theta_{3}^{4} \theta_{2}^{3} \theta_{1}- \\
& -36 \theta_{2} \theta_{1}^{6} \theta_{3}+96 \theta_{1}^{3} \theta_{2}^{3} \theta_{3}^{2}+80 \theta_{1}^{3} \theta_{2}^{4} \theta_{3}-3 \theta_{1}^{4} \theta_{3}^{4}-12 \theta_{1}^{5} \theta_{3}^{3}-18 \theta_{1}^{6} \theta_{3}^{2}+48 \theta_{2}^{3} \theta_{1}^{5}- \\
& -48 \theta_{2}^{5} \theta_{1}^{3}-12 \theta_{2} \theta_{1}^{7}+40 \theta_{1}^{4} \theta_{2}^{4}-64 \theta_{1}^{2} \theta_{2}^{6}-16 \theta_{2}^{5} \theta_{3}^{3}-12 \theta_{1}^{7} \theta_{3} .
\end{aligned}
$$

Рассмотрим различные случаи.

1. Пусть $\theta_{3}=\theta_{1}+1$ и $\theta_{2}=1$, тогда

$$
\begin{gathered}
B=4 \theta_{1}^{4}+24 \theta_{1}^{3}+41 \theta_{1}^{2}+20 \theta_{1}-2, \\
D=-144 \theta_{1}+344 \theta_{1}^{3}-156 \theta_{1}^{2}+541 \theta_{1}^{4}-48 \theta_{1}^{8}-256 \theta_{1}^{7}-424 \theta_{1}^{6}-32 \theta_{1}^{5} .
\end{gathered}
$$

Можно факторизовать $D$ следующим образом:

$$
D=-\theta_{1}\left(4+3 \theta_{1}\right)\left(2 \theta_{1}+3\right)^{2}\left(2 \theta_{1}^{2}+\theta_{1}-2\right)^{2} .
$$

Тогда $D<0$, т. е. все 2 -периодические меры Гиббса трансляционно-инвариантны.

2. Пусть $\theta_{3}=\theta_{1}$ и $\theta_{2}=1$, тогда

$$
\begin{gathered}
B=4 \theta_{1}^{4}+20 \theta_{1}^{3}+16 \theta_{1}^{2}-4, \\
D=-48 \theta_{1}^{8}-160 \theta_{1}^{7}+160 \theta_{1}^{4}+320 \theta_{1}^{5}-160 \theta_{1}^{3}-128 \theta_{1}^{2}+16 .
\end{gathered}
$$

Можно факторизовать $D$ следующим образом:

$$
D=-16\left(3 \theta_{1}^{4}+10 \theta_{1}^{3}+6 \theta_{1}^{2}-1\right)\left(\theta_{1}-1\right)^{2}\left(\theta_{1}+1\right)^{2} .
$$

Используя программу MAPLE, мы нашли, что уравнение $D=0$ имеет два действительных корня, один из которых положителен, $\theta_{D} \approx 0.323591553488076$. Следовательно, если $\theta_{1}>\theta_{D}$, то $D<0$, т. е. все 2-периодические меры Гиббса трансляционно-инвариантны. Если $0<\theta_{1}<\theta_{D}$, то $D>0$ и $B<0$ (см. рис. 8), что влечёт существование 2-периодической меры Гиббса. Получаем следующий результат.

Теорема 9. Пусть $\theta_{3}=\theta_{1} u \theta_{2}=1$. Если $0<\theta_{1}<\theta_{D}$, mо имеет место фазовый переход.

ЗАМЕЧАНИЕ 2. При выполнении условий теоремы 9 , если $\theta_{1}>3$, можно найти трансляционно-инвариантную меру Гиббса. Теорема 9 говорит о том, что существование 2-периодической меры Гиббса не влечёт за собой существование трансляционно-инвариантной меры Гиббса. 


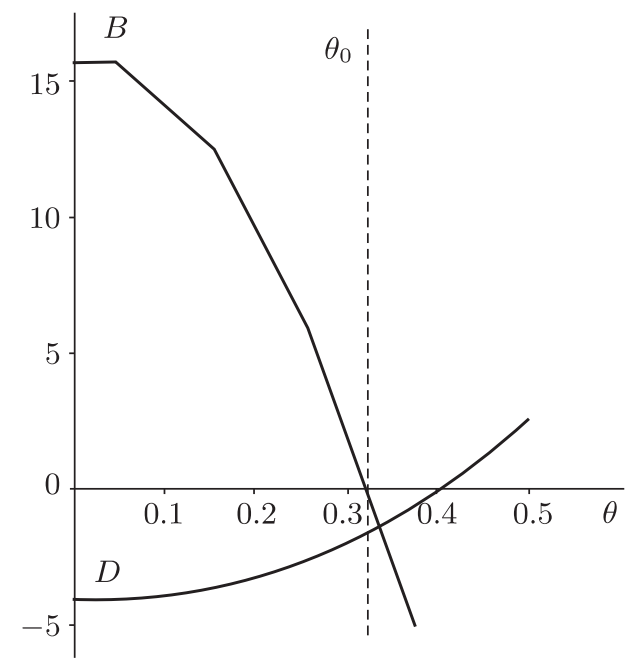

Рис. 8. Существование 2-периодического решения (случай 2).

3. Пусть $\theta_{1}=\theta_{2}$ и $\theta_{3}-$ произвольное число, тогда

$$
\begin{gathered}
B=7 \theta_{1}^{2} \theta_{3}^{2}+11 \theta_{1}^{4}+16 \theta_{3} \theta_{1}^{3}+2 \theta_{1} \theta_{3}^{3}, \\
D=-7 \theta_{1}^{4} \theta_{3}^{4}+22 \theta_{1}^{6} \theta_{3}^{2}-4 \theta_{1}^{5} \theta_{3}^{3}-4 \theta_{1}^{3} \theta_{3}^{5}-23 \theta_{1}^{8}+16 \theta_{3}^{7} \theta_{3} .
\end{gathered}
$$

Можно факторизовать $D$ следующим образом:

$$
D=-\theta_{1}^{3}\left(23 \theta_{1}^{3}+30 \theta_{1}^{2} \theta_{3}+15 \theta_{1} \theta_{3}^{2}+4 \theta_{3}^{3}\right)\left(-\theta_{3}+\theta_{1}\right)^{2} .
$$

Следовательно, $D<0$ для любых $\theta_{1}, \theta_{3}>0$, т. е. все 2-периодические меры Гиббса являются трансляционно-инвариантными.

\section{Список литературы}

[1] Р. Бэкстер, Точно решаемые модели в статистической механике, Мир, М., 1985.

[2] Х.-О. Георги, Гиббсовские меры и фазовые переходь, Мир, М., 1992.

[3] M. C. Marques, "Three-state Potts model with antiferromagnetic interactions: a MFRG approach", J. Phys. A: Math. Gen., 21:4 (1988), 1061-1068.

[4] M.P. Nightingale, M. Schick, "Three-state square lattice Potts antiferromagnet", J. Phys. A: Math. Gen., 15:1 (1982), L39-L42.

[5] R. B. Potts, "Some generalized order-disorder transformations", Proc. Cambridge Philos. Soc., 48:1 (1952), 106-109.

[6] F. Y. Wu, "The Potts model", Rev. Modern Phys., 54:1 (1982), 235-268.

[7] S. N. Dorogovtsev, A. V. Goltsev, J.F.F. Mendes, "Potts model on complex networks", Eur. Phys. J. B, 38:2 (2004), 177-182.

[8] F. Peruggi, "Probability measures and Hamiltonian models on Bethe lattices. I. Properties and construction of MRT probability measures", J. Math. Phys., 25:11 (1984), 3303-3315.

[9] F. Peruggi, "Probability measures and Hamiltonian models on Bethe lattices. II. The solution of thermal and configurational problems", J. Math. Phys., 25:1 (1984), 3316-3323.

[10] П. Н. Тимонин, "Индуцированные неоднородностями фазовые переходы второго рода в модели Поттса на иерархических решетках", ЖКЭТФ, 126:5 (2004), 1198-1208. 
[11] F. Peruggi, F. di Liberto, G. Monroy, "Potts model on Bethe lattices. I. General results", J. Phys. A: Math. Gen., 16:4 (1983), 811-827.

[12] F. Peruggi, F. di Liberto, G. Monroy, "Phase diagrams of the $q$-state Potts model on Bethe lattices", Phys. A, 141:1 (1987), 151-186.

[13] Н. Н. Ганиходжаев, "О чистых фазах ферромагнитной модели Поттса с тремя состояниями на решетке Бете второго порядка", ТМФ, 85:2 (1990), 163-175.

[14] N. N. Ganikhodjaev, F. M. Mukhamedov, J. F. F. Mendes, "On the three state Potts model with competing interactions on the Bethe lattice", J. Stat. Mech., 2006:8 (2006), P08012, $29 \mathrm{pp}$.

[15] К. Престон, Гиббсовские состояния на счетных множествах, Мир, М., 1977.

[16] N. N. Ganikhodjaev, U. A. Rozikov, "On disordered phase in the ferromagnetic Potts model on the Bethe lattice", Osaka J. Math., 37:2 (2000), 373-383.

[17] У.А. Розиков, Р. М. Хакимов, "Периодические меры Гиббса для модели Поттса на дереве Кэли", ТМФ, 175:2 (2013), 300-312.

[18] F. M. Mukhamedov, "On a factor associated with the unordered phase of $\lambda$-model on a Cayley tree", Rep. Math. Phys., 53:1 (2004), 1-18.

[19] У.А. Розиков, "Описание предельных гиббсовских мер для $\lambda$-моделей на решетках Бете", Сиб. матем. журн., 39:2 (1998), 427-435.

[20] U. A. Rozikov, Gibbs Measures on Cayley Trees, World Sci., Singapore, 2013.

[21] Ф. М. Мухамедов, У. А. Розиков, “Экстремальность неупорядоченной фазы неоднородной модели Поттса на дереве Кэли", ТМФ, 124:3 (2000), 410-418. 